\title{
The analytical presentation of solid border basement in conditions of solid stamp effect
}

\author{
Gevorg Ter-Petrosyan* \\ National University of Architecture and Construction, Teryan 105 st., Yerevan 009, Armenia
}

\begin{abstract}
The current level of construction and, moreover, the future value the right apressiation of mechanical capabilities of the structure basement. The calculation of additional resource of strength basement of each structure brings to increasing of value of structure. The increasing of reliability and sustainability of structures is one of the problems of modern applied mechanics. The correct assessment of the strength of basement is very important in solving this problem. It is still conditional to determine the computational depth of basement. Numerous experimental studies have shown that in the case of distributed load of constant intensity, the dependence of sediment from the base sizes is expressed by strict nonlinear connection. The solvation of problem of constant thick layer gives the linear connection by methods of elasticity of theory. The purpose of eliminating that essential discrepancy with experimental results, the solved problem, proposed by the academician S.S. Grigoyan and applied by his students, is an important step towards solving.
\end{abstract}

\section{Introduction}

For projecting the bases of constructions you must select the depth of elastic sphere of its base.

The experimental studies have shown $[1,2,3,4]$ that the depth of the elastic layer of the basement effecting on it from greatness of load and sizes of bases, depends on non-linear link. The solvation of the problem by method of elasticity gives the linear dependence.

For the purpose of elimination of discrepancy with experimental data, the academician S.S. Grigoryan's suggestion [5] concerning the model of solid material, was the fundamental step in the correct assessment work of discrepancy of noted disagreement and the mechanical chances of basement.

\section{Methods}

The correct selection of basement in the problems of bases calculation is the most important precondition. It is supposed that the material of basement in conditions of affection of its gravity forces on a certain density is already state of solidity. It means that additional deformations don't emerge in domain of solid material (Fig. 1).

\footnotetext{
* Corresponding author: terpetrosyan47@bk.ru
} 


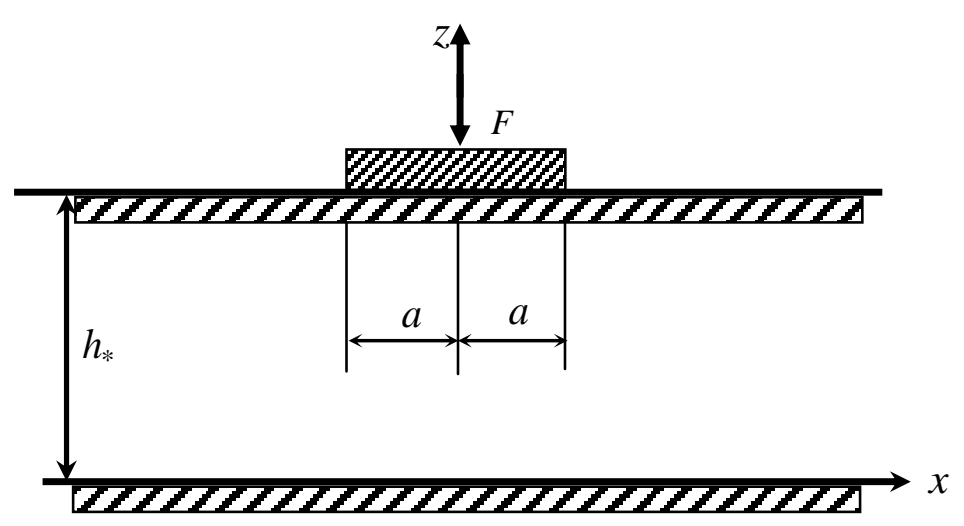

Fig. 1. The plain problem of half-distance with solid material under the effect of solid stamp.

We have the following plain problem with boundary conditions;

$$
\begin{aligned}
& w=S \text { when }|x| \leq a \\
& \sigma_{z z}=O \text { when }|\mathrm{x}|>\mathrm{a}
\end{aligned}
$$

on the edge of elastic and solid domain

$$
u=w=0
$$

Here $\mathrm{u}$ and $\mathrm{w}$ are marked to point the replacements in $\mathrm{x}$ and $\mathrm{z}$ directions, $\mathrm{S}$ - is the sediment of stamp. Such problem set like this, is strictly non-linear because the solid edge on which the edge conditions are put, is preliminary unknown and is determined during the solution of the problem. The $h *$ depth of solidity from the weight of the partition is determined by the following formula:

$$
h *=3 \frac{1}{1+} \quad \frac{\sigma *}{\gamma},
$$

where $\sigma *-$ is the value of critical comprehensive pressure, $\vartheta$-is the material coefficient of Puason. The problem set like this, is solved by us with the method of [6] local variations for which all the magnitudes are expressed with immeasurable views.

$$
\begin{array}{r}
\chi=\frac{x}{h *}, \quad z=\frac{x}{h *}, U=\frac{u E}{\sigma * h *}, W=\frac{w E}{\sigma * h *}, \\
\varepsilon_{x x}=\frac{E \varepsilon_{x x}}{\sigma *}, \quad \varepsilon_{x z}=\frac{E \varepsilon_{x z}}{\sigma *}, \quad \varepsilon_{z z}=\frac{E \varepsilon_{z z}}{\sigma *} \\
\Sigma_{x x}=\frac{\sigma_{x x}}{\sigma *}, \quad \Sigma_{x z}=\frac{\sigma_{x z}}{\sigma *}, \quad \sum_{z z}=\frac{\sigma_{z z}}{\sigma_{*}}:
\end{array}
$$

E- is the elastic module of the material. The solvation of the problem is realized by application of minimum principle. 


$$
\ni=0,5 \iint_{D}\left(\delta \sigma_{x x} \delta \varepsilon_{x x}+2 \delta \sigma_{x z} \cdot \delta \varepsilon_{x z}+\delta \sigma_{z z} \cdot \delta \varepsilon_{z z}\right) d x d z
$$

Here $\delta \sigma_{x x}, \delta \sigma_{x z}, \delta \sigma_{z z}$-are the increases of tensions, $\delta \varepsilon_{x x}, \delta \varepsilon_{x z}, \delta \varepsilon_{z z}$-are the increases of deformations and $\mathrm{D}$ - is the elastic domain. Expressing the tension increases from deformation increases and inserting (4) we get,

where,

$$
\ni=0,5 \int\left[a\left(\varphi_{x}^{2}+\psi_{z}^{2}\right)+2 b \varphi_{x} \psi_{z}+\frac{1}{2} c\left(\varphi_{z}+\psi_{x}\right)^{2}\right] d x d z
$$

$$
a=\frac{1-\vartheta}{(1+\vartheta)(1-2 \vartheta)}, \quad b=\frac{\vartheta}{(1+\vartheta)(1-2 \vartheta)}, \quad c=\frac{1}{1+\vartheta}
$$

The solvation of the problem by unmeasurable data, we have received numerical data expressing the sediment of hard stamp and the variation of border line of hard material, the values of which on the symmetry $\mathrm{Z}$ axis are listed in the table [6] ;

Table 1. The changes of the solidity of edge.

\begin{tabular}{|c|c|c|c|c|c|c|c|c|}
\hline $\mathbf{X}$ & 0.0 & 0.2 & 0.4 & 0.6 & 0.8 & 1.0 & 1.2 & 1.4 \\
\hline \multicolumn{1}{|c|}{} & 0.110 & 0.095 & 0.071 & 0.045 & 0.032 & 0.016 & 0.010 & 0.010 \\
\hline 0.4 & 0.241 & 0.232 & 0.153 & 0.092 & 0.040 & 0.020 & 0.012 & 0.011 \\
\hline 0.55 & 0.342 & 0.321 & 0.215 & 0.112 & 0.051 & 0.025 & 0.015 & 0.012 \\
\hline 0.65 & 0.435 & 0.392 & 0.265 & 0.142 & 0.075 & 0.034 & 0.018 & 0.013 \\
\hline 0.75 & 0.551 & 0.495 & 0.320 & 0.164 & 0.082 & 0.041 & 0.021 & 0.018 \\
\hline 0.80 & 0.715 & 0.631 & 0.372 & 0.171 & 0.091 & 0.045 & 0.025 & 0.019 \\
\hline
\end{tabular}

We have chosen the graph of hard stamp and the dependence of solidity edge;

$$
\Gamma=b \quad e^{(\alpha x)^{2}}
$$

the function which $\alpha$ and $b$ must be determined.

In order to be consistent with the choice of the function $\Gamma$, for the purpose of using the average method, first of all check the circumstance to be related with $x \lg x$ and $\Delta \ell g x$ dependence linearity. Calculate these values. The calculation is presented in the following table (Table 2);

Table 2. The process of calculation of average method of application.

\begin{tabular}{|c|c|c|c|c|c|c|c|c|}
\hline $\mathrm{X}$ & 0.0 & 0.2 & 0.4 & 0.6 & 0.8 & 1.0 & 1.2 & 1.4 \\
\hline$\ell g \Gamma$ & & -0.699 & -0.398 & -0.222 & -0.017 & 0.000 & 0.079 & 0.146 \\
\hline$\Delta \ell g \Gamma$ & & 0.301 & 0.176 & 0.205 & 0.017 & 0.079 & 0.067 & \\
\hline$\Gamma(\mathrm{w}=0.2)$ & 0.110 & 0.095 & 0.071 & 0.045 & 0.032 & 0.016 & 0.012 & 0.010 \\
\hline$\ell g \Gamma$ & -0.959 & -1.022 & -1.149 & -1.347 & -1.495 & -1.799 & -1.921 & -2.000 \\
\hline$\Delta \ell g \Gamma$ & -0.063 & -0.127 & -0.198 & -0.148 & -0.304 & -0.125 & -0.079 & \\
\hline
\end{tabular}


Constructing the graph of $\Delta \ell g x$ and $\Delta \ell g \Gamma$ dependency, we notice that it is close enough to the straight line. It follows from this, that the dependence $\Gamma$ can be presented by the following aspect (8). The $\alpha$ and $b$ constants are determined by the average method. We make the initial equations.

$$
\ell g \Gamma_{i}=\ell g b \quad\left(a x_{i}\right)^{2} \quad \ell g e,
$$

which being grouped in two groups, adding member by member, we get ;

$$
2\left\{\begin{array}{l}
-3,518=3 \lg b-\alpha^{2} \cdot 0,2432 \\
-5,215=3 \lg b-\alpha^{2} \cdot 1,3376
\end{array}\right.
$$

the latest system solution we get ;

$$
\alpha=1,2452, b=0,0893 \text { : }
$$

So, the function of solidity will have the following aspect;

$$
\Gamma=0,083 \quad e^{(1,2452 x)^{2}}
$$

In order to be able to apply the received (12) formula in cases of different magnitudes of the sediment stamp, we insert the $\beta$ coefficient.

Let's determine those coefficient values on the symmetry axe by means of getting the values of changes of solid border by variation method.

For determination of $\beta$ we get (Table 1).

Table 3. The coordinates of solid limit on the $r$ axis.

\begin{tabular}{|c|c|c|c|c|c|c|}
\hline $\mathrm{S}$ & 0.2 & 0,55 & 0.4 & 0,65 & 0.75 & 0.80 \\
\hline$\beta$ & 6.1591 & 6.9632 & 6.7469 & 7.4942 & 8.2867 & 10,0084 \\
\hline
\end{tabular}

For determination of $\beta$ we get (Table 4).

Table 4. Table of determination of $\beta$ coefficient.

\begin{tabular}{|c|c|c|c|c|c|c|}
\hline $\mathrm{S}$ & 0.2 & 0.4 & 0,55 & 0,65 & 0.75 & 0.80 \\
\hline$\beta$ & 6.1591 & 6.7469 & 6.9632 & 7.4942 & 8.2867 & 10,0084 \\
\hline
\end{tabular}

So, the function of solid edge, we get the following final aspect.

$\Gamma=\beta \cdot 0,0893 \cdot e^{-(1,2452 x)^{2}} \cdot S$

In cases when the s value of the sediment of sediment stamp is absent in the table, so the value of $\beta$ corresponding to given size we determine by means of nearby values of interpolation. 


\section{Conclusions}

For the purpose of verifying the reliability of the (13) formula for determining the solid border of the base material, we have compared the values of having [6] local variation method and (13) calculated by formula.

We have done the comparison on the $\mathrm{r}$ axis of symmetry by means of replacement of solid edge (table). The comparison shows, that on the symmetric axis of stamp (13), the differences of replacement of the edge of solid material is being determined by method of formula and local variations are very small, and are negligible in the point of practical application. Therefore, (13) the formula can be used for the ribbon base of constructions, in order to receive the determination of initial size of the depth of deformative layer of basement.

Table 5. The comparison of modification of edge of solidity.

\begin{tabular}{|c|c|c|c|c|c|c|}
\hline $\mathrm{S}$ & 0.2 & 0.4 & 0.55 & 0.65 & 0.75 & 0.8 \\
\hline $\begin{array}{c}\Gamma \text { variation } \\
\text { method }\end{array}$ & 0,110 & 0.241 & 0.342 & 0.435 & 0.551 & 0.715 \\
\hline $\begin{array}{c}\Gamma(13) \\
\text { by formula }\end{array}$ & 0,1100 & 0.24099 & 0.34199 & 0.4330 & 0.5509 & 0.71500 \\
\hline$\Delta$ & 0,0000 & 0.00001 & 0.00001 & 0.00000 & 0.00001 & 0.0000 \\
\hline
\end{tabular}

\section{References}

1. S.S Vyalov, J. Foundations and soil mechanics, No.3, 1966

2. M.I. Gorbunov-Posadov, J. The current state of the scientific foundations of foundation engineering, 1967

3. S.S Grigoryan, J. Mechanical Engineer of the MSU, No.2, 1974

4. K..E. Egurov, J. Repots of the AS, V.133, No.4, 1960

5. S.S. Grigoryan, Mechanics of the whole range and problems of analysis, 1972

6. G.V. Ter-Petrosyan, J. Reports of AS Arm. SSR, V. 61, No.5, 1975

7. S.S. Grigoryan, G.V. Ter-Petrosyan, J. Materials of the 32 nd R. Scientific-Technical Conference, Part 2, 2003

8. F.L. Chernousko, N.V. Balinchuk, Variational tasks engineer and management, 1973

9. G.V. Ter-Petrosyan, J. Repots of AS Arm SSR, Science of ground, No.1, 1976

10. I.N. Bronshteyn, K..A.. Semendyaev, Math handbook, 1981

11. G.V. Ter-Petrosyan, J. Proceedings of the $10^{\text {th }}$ International Conference on Contemporary Problems of Architecture and Construction, 2018 